\title{
Forecasting Macroeconomic Labour Market Flows What Can We Learn from Micro-level Analysis?
}

Wilke, Ralf

Document Version

Accepted author manuscript

Published in:

Oxford Bulletin of Economics and Statistics

DOI:

10.1111/obes.12222

Publication date:

2018

License

Unspecified

Citation for published version (APA):

Wilke, R. (2018). Forecasting Macroeconomic Labour Market Flows: What Can We Learn from Micro-level Analysis? Oxford Bulletin of Economics and Statistics, 80(4), 822-842. https://doi.org/10.1111/obes.12222

Link to publication in CBS Research Portal

\section{General rights}

Copyright and moral rights for the publications made accessible in the public portal are retained by the authors and/or other copyright owners and it is a condition of accessing publications that users recognise and abide by the legal requirements associated with these rights.

Take down policy

If you believe that this document breaches copyright please contact us (research.lib@cbs.dk) providing details, and we will remove access to the work immediately and investigate your claim. 


\title{
Forecasting Macroeconomic Labour Market Flows: What Can We Learn from Micro-level Analysis? \\ Ralf Wilke
}

Journal article (Accepted version)

\begin{abstract}
This is the peer reviewed version of the following article: Forecasting Macroeconomic Labour Market Flows : What Can We Learn from Micro-level Analysis? / Wilke, Ralf. In: Oxford Bulletin of Economics and Statistics, 17.11.2017., which has been published in final form at http://dx.doi.org/10.11l1/obes.12222.
\end{abstract}

This article may be used for non-commercial purposes in accordance with Wiley Terms and Conditions for Self-Archiving.

Uploaded to Research@CBS: December २017 


\title{
Forecasting macroeconomic labour market flows: What can we learn from micro level analysis?*
}

\author{
Ralf A. Wilke ${ }^{\dagger}$
}

October 2017

\begin{abstract}
Forecasting labour market flows is important for budgeting and decision making in government departments and public administration. Macroeconomic forecasts are normally obtained from time series data. In this paper we follow another approach that uses individual level statistical analysis to predict the number of exits out of unemployment insurance claims. We present a comparative study of econometric, actuarial and statistical methodologies that base on different data structures. The results with records of the German unemployment insurance suggest that prediction based on individual level statistical duration analysis constitutes an interesting alternative to aggregate data based forecasting. In particular forecasts of up to 6 months ahead are surprisingly precise and are found to be more precise than considered time series forecasts.
\end{abstract}

Keywords: unemployment insurance, in-sample forecasting, duration analysis JEL: C53, C55, J60

${ }^{*}$ Thanks are due to Jens P. Nielsen, Lola Martínez, Héctor C. Villanova and Enzo Weber for helpful discussions and to the former two for making their $\mathrm{R}$ code for the chain ladder model (-to be released as package "Double Chain Ladder"-) available. The comments of two reviewers haven been also gratefully acknowledged.

${ }^{\dagger}$ Copenhagen Business School, Department of Economics, Porcelænshaven 16A, 2000 Frederiksberg, Denmark, E-mail: rw.eco@cbs.dk 


\section{Introduction}

Labour market analysis on the individual level normally uses individual level data and macro level analysis bases on aggregate data (see for example Barnichon and Nekarda, 2012). Until recently such a separation was natural due to restrictions on data availability and computing performance. Although, surveys, such as the labour force survey, are frequently used to validate or construct macroeconomic figures such as the unemployment rate or labour market flows (see for example Elsby et al., 2015, Hutter and Weber, 2015), econometric forecasting of macroeconomic figures is typically based on aggregate data. Examples include Brown and Moshiri (2004), Sermpinis et al. (2014) and Hutter and Weber (2017). The increased availability of large linked administrative individual data opens up new opportunities for micro level statistical analysis as information on individual level is becoming available for the population. While these data have become the industry standard for applied analysis on individual level, little efforts have been devoted to link the statistical analysis on individual level with forecasts for the macro level.

Economic literature on linking microeconometric analysis with macro models is sparse. Partly this is done in microsimulation models. However, these models are less a statistical approach but more a computational tool in applied economics. Their core is simulating parts of the economy using models based on economic theory. More related to the goals of this study, new research in actuarial sciences has developed forecasts for aggregate figures on the grounds of micro data level analysis (compare Antonio and Plat, 2014). This approach has also been shown to be useful in estimating outstanding claim liabilities in the disability insurance (Spierdijk and Koning, 2014) and forecasting mesothelioma mortality (Martínez-Miranda et al., 2015). This paper adopts 
the idea of using individual level data to construct forecasts for macroeconomic figures. The considered models exploit the richness of the data and avoid parametric restrictions on the micro level as much as possible. An empirical study is presented which compares forecasts obtained by different approaches including a classical time series forecast. Extensive unemployment insurance claim records from Germany are used to predict the number of unemployment benefits leavers. Within this application it is shown how macroeconomic labour market forecasts are obtained from estimated individual level transition probabilities. The latter are estimated using data about individual employment biographies in past periods. By focussing on exits out of the unemployment insurance, we consider a standard problem in actuarial sciences, the so-called reserving problem. There the insurer uses data on past claims and contract signing dates to produce a forecast for future insurance claims or outstanding liabilities. Using a simple data structure in form of a triangle, these so-called Chain Ladder Method (CLM) (compare Weindorfer, 2012) are used by most if not all insurers to estimate outstanding liabilities. Thus, these estimates are very important for the decision about the adequate size of financial reserves. The statistical properties of these actuarial methods are well developed (Kuang et al., 2009, Pigeon et al., 2013). Structured density forecasting is adopted in this study as it uses individual level data to construct in-sample forecasts for the aggregate. In contrast to time series models these forecasts do not simply extrapolate an aggregate figure but use as much as possible individual level information at the edge of the observation period to construct the forecast. As third contender, statistical duration models are used to construct macro level forecasts. The idea here is to estimate individual level probabilities for existing unemployment insurance claims. Thus, this work puts an interdisciplinary method mix to data to analyse the same problem and to explore how estimation results compare. A rather non-technical presentation of the material is chosen to make the material attractive for practitioners. 
The paper is structured as follows. Section 2 briefly presents the economic and institutional framework for the empirical analysis. Section 3 provides details about the administrative data used in the analysis. Section 4 describes the methodologies and Section 5 presents the forecasting results. Section 6 summarises the main findings and outlines trajectories for further improvements.

\section{Unemployment insurance in Germany}

Precise prediction of the number of unemployment benefit leavers is not only important for policy makers to be able to build reliable expectations about state of the labour market but also for financial planning units within the unemployment insurance. In Germany, the unemployment insurance is administered by the Federal Employment Agency (FEA), located at Nuremberg. Unemployed have an entitlement for contribution based unemployment benefits if they were previously employed and paid contributions to the national social insurance, of which the unemployment insurance is a part. The length of the entitlement for unemployment benefits depends mainly on how long the unemployed has previously paid in, on their age and to some extent on the business sector and the calendar year. Maximum entitlement lengths are 12 months for younger individuals, while maximum entitlement length for older unemployed have been up to 32 months for most of the observation period of this study. Once unemployment benefits are exhausted, the unemployed may be entitled to means tested unemployment assistance. Thus, each unemployed claiming unemployment benefits will exit from these benefits at the latest when the entitlements are exhausted. For more details about the German unemployment compensation system in the relevant period see Plaßmann (2002). The main analysis period of this paper is 2002-2005 to construct a forecast for 2006. It is worth mentioning that the en- 
titlement length for unemployment benefits (in particular maximum entitlement lengths) were broadly unchanged during the period 2002-2005. But there were significant changes in entitlement lengths before and after this period. One policy change in 1997, which became gradually effective during the period 1999-2001, increased requirements for extended entitlement lengths ( $>12$ months). Another policy change led to a reduction in maximum entitlement lengths from 32 to 18 months from February 2006 and another change increased it back to 24 months from January 2008. This study therefore focuses on period with stability in entitlements, although many other labour market regulations were subject to change during this period.

Looking at outflows of unemployment benefit claimants is not the same as looking at outflows of all unemployed but only the former are of interest for the unemployment insurance. The FEA has confirmed that projections of benefit counts, inflows, and outflows are important for their liquidity management and budgeting. In their current internal processes they construct projections on the grounds of current year-to-year changes in aggregate figures. Therefore, a comparison of various methods, as in this paper, is highly relevant to them and practitioners in other unemployment insurances.

\section{Data}

The analysis of this paper is based on linked administrative individual data from Germany. In particular it uses the Sample of Integrated Labour Market Biographies (SIAB, Scientific Use File) of the Institute for Employment Research (IAB), the research institute of the FEA. See vom Berge et al. (2014) for more details on these data. The SIAB is a $2 \%$ random sample of the German population which is contributing to the national social insurance. It comprises of daily 
administrative records for the period 1975-2010. Among linked administrative records from various sources, the data comprise claim periods of unemployment benefits. For the analysis, these subsequent records for the same individual are linked to claim spells if the interruption in the claim was less than 30 days and if there was no other administrative record pointing to some other economic activity during this period. These short interruptions are therefore mainly due to short periods of unavailability or benefit sanctions. After unemployment benefits claim periods have been constructed, they are converted from days to months and all subsequent analysis is conducted on monthly basis. Given that most individuals in the data do not have a claim spell and others have more than one claim spell, the resulting number of claim spells is much smaller than the number of individuals in the SIAB. From the SIAB it is also directly possible to construct aggregate macro figures, such as the number of transitions into and out of unemployment benefits by simply counting the transitions at specific time points.

The main analysis focuses on the period 2002-2006, which we denote as monthly periods $t=1, \ldots, 60$, where $t=1$ corresponds to January 2002 and greater $t$ to subsequent months. Estimation uses data from 2002-2005, i.e. from periods $t=1, \ldots, 48$, to construct monthly forecasts for the number of benefit leavers in $2006(t>48)$. We denote the number of claimants stopping to claim in $t$ as $O_{t}$ for all $t$ and any forecast as $O_{t}^{f o r e c a s t}$. $O_{t}$ is extracted for all $t$. All individual benefit claim durations that commence in the period 2002-2005 are computed which results in $i=1, \ldots, 364,362$ individual claim durations $l_{i} \in\{1, \ldots, L\} . L$ is the maximum claim duration, which is 32 months or possibly a couple of months longer in our application due to the legal restriction on the maximum benefit entitlement lengths. If a duration is not completed by the end of 2005 it is marked as censored. From these durations it is possible to determine elapsed claim duration at each period $t$ for those who are claiming benefits in $t$. This 
is denoted as $l_{i}(t)$ for all $i$ that claim benefits in $t$. The individual level analysis model also requires information about inflows- this is individuals who start a claim duration- and this is

denoted by $I_{t}$ and any forecast by $I_{t}^{\text {forecast }}$, respectively. Thus, $I_{t}$ is also extracted for all $t$.

The data preparation code is made available as supplementary material but access to the SIAB requires a valid data use agreement with the Research data centre of the FEA (fdz.iab.de). Researchers who are only interested in running and understanding the code may consider working with the campus file version of these data. Access details are provided in the supplementary material.

The analysis has been repeated for other periods to forecast the years 2002, 2003, 2004, 2005 and 2009 and these results are presented in the supplementary material to this paper. A similar analysis could be also conducted to predict outflows out of registered unemployment. This would, however, require a slightly different data preparation using job seeking records in the individual level data instead of benefit claim periods.

\section{Forecasting Benefit Outflows}

The starting point is a simple time series regression with seasonality and trend using aggregate data for 48 months (Jan 2002-Dec 2005). We consider the following models for benefit claim outflows and inflows:

$$
\begin{aligned}
O_{t} & =\beta_{0}+\sum_{m=2}^{12} \theta_{m} d_{m}+\beta_{1} t+\beta_{2} t^{2}+u_{t} \\
I_{t} & =\alpha_{0}+\sum_{m=2}^{12} \omega_{m} d_{m}+\alpha_{1} t+\alpha_{2} t^{2}+v_{t}
\end{aligned}
$$


for $t=1, \ldots, 48 . d_{m}$ are calendar month dummies, $t$ is the trend (taking on the value of the period) and $u_{t}$ and $v_{t}$ are the unobserved error terms. OLS is used for these models to estimate the unknown $\beta_{j}, \theta_{j}, \alpha_{j}$ and $\omega_{j}$. The Newey-West procedure (Newey and West, 1987) is used to obtain autocorrelation robust standard errors. Other polynomial specifications of the trend component in the time series models have been also used. Results were somehow sensitive to this but the main result pattern were invariant. The use of autoregressive terms did not markedly improve the model fit and for reasons of simplicity these results have not been reported. After estimating these models, $O_{t}$ and $I_{t}$ are forecasted using future values of $t$ and by choosing the appropriate calender month $d_{m}$. These forecasts are denoted as $O_{t}^{T S}$ and $I_{t}^{T S}$ and are computed for all calendar months in the year 2006. An intercept corrected forecast for $O_{t}$ is also obtained by adding the last in-sample residual to $O_{t}^{T S}$. The resulting forecasts for $O_{t}$ are then compared with the observed data as well as several micro analysis based forecasts for the year 2006 .

The general idea to forecast labour market flows based on micro level estimates is based on what is known as reserving in actuarial sciences. Here insurance firms use individual claims data about the date of the claim and the contract sign date to construct estimates for outstanding liabilities in future periods (compare Pigeon et al., 2014). This involves estimating densities for the intensity (distribution of new contract signing dates) and the density for the development times (time between signing the new contract and the occurrence of the claim). In our problem the latter corresponds to estimating the distribution of the length of unemployment claim periods and the former to estimating the distribution of new entries into unemployment benefits. Insurance firms normally organise data in form of a triangle and use the so called chain ladder method (CLM) to estimate the distributions. For instance, see Weindorfer (2012) for an informal introduction. These methods are popular because they rely on semi-aggregated 
data with minimal requirements on the availability of individual level data. Compare Appendix A.I for more details and a statistical representation of this approach to in-sample forecasting. In statistics it is also referred to as structured density forecasting. There is a growing literature in actuarial sciences suggesting various extensions or alternatives to the classical chain ladder method. Kuang et al. (2011) propose an extended chain ladder model. Mammen et el. (2015) and Hiabu et al. (2014) re-phrase the problem to a structured density forecasting framework. Lee et al. (2015) incorporate an additional seasonality component. While these structure density forecasting models are nonparametric and involve Kernel smoothing, Pigeon et al. (2013) and Pigeon et al. (2014) develop alternative approaches by parameterizing distributions and employing maximum likelihood estimation. We follow here the spirit of nonparametric models in order to avoid assumptions as much as possible. Similar to Antonio and Plat (2014) this paper presents approaches to make use of micro level analysis as much as possible.

Estimation results for various models are compared. The discussion of the results is mainly restricted to point estimates but exemplary confidence intervals and mean squared errors are also reported.

The forecast for the total number of claim outflows is the sum of forecasts A and B:

(A) Forecasting the future outflows of those who are in stock today (i.e. those who are claiming insurance benefits at the end of 2005).

(B) Forecasting future outflows of those who will start a claim period during the forecasting period (i.e. those who start claiming insurance benefits sometime in 2006). 
Forecast A Four forecasts A are computed: The CLM is used along with three alternative approaches that utilise estimated transition probabilities (obtained by CLM and conventional duration models) and information about the stock of benefit claimants at the end of 2005 . These forecasts are directly comparable and reveal how different data and model restrictions lead to different results. The first forecast is obtained by the classical CLM, which is described in Appendix AI, and denoted as $O_{t}^{C L M(A)}$. This forecast is based on a data triangle, which contains observed counts of benefit exits by calender month and by benefit duration. For more details see Appendix AI, where the data triangle of this application is shown in Figure 4. The CLM forecast uses $f^{C L}(l)$, the CLM estimate for the density of claim duration $l$ which is given in Equation (6) in the Appendix. The density representation of the CLM is presented in greater detail in Martínez-Miranda et al. (2013). The second forecast, $O_{t}^{C L(A)}$, is a hybrid of the CLM and a duration model. It uses $f^{C L}(l)$ but instead of the estimated density of benefit inflows, it uses data on the stock of claimants at the end of 2005 along with information about their length of claim periods at the end of 2005. This information can be organised as a $L \times 1$ vector. Let $i=1, \ldots, N_{t 0}$ be the benefit claimants in $\mathrm{t} 0=\mathrm{Dec} 2005$ (or $\mathrm{t}=48$ ). The forecast is constructed as follows:

$$
O_{t}^{C L(A)}=\sum_{i=1}^{N_{t 0}} c f^{C L}\left(l_{i}(t)\right),
$$

where $c f^{C L}\left(l_{i}(t)\right)$ is the conditional density of exiting benefits in period $t=49, \ldots, 60$ given that the unemployed is claiming unemployment benefits in $t 0$ and has a claim duration of $l_{i}(t)$ in period $t$. This is $c f^{C L}(l)=f^{C L}(l) / \int_{l}^{\infty} f^{C L}(u) d u$. The conditional density is used to make sure that the sum of probabilities taken over future claim months is one. The difference between $O_{t}^{C L M(A)}$ and $O_{t}^{C L(A)}$ is therefore that the former estimate only uses information about the number of completed claim spells in the past to estimate the stock of current claimants, while the latter uses the actual number of claimants at the end of 2005. 
The third forecast also uses the stock of claimants at the end of 2005 but different estimated densities of claim duration. The classical chain ladder model makes the implicit assumption that there is no seasonality in the distribution of claim duration. This is that the distribution of duration is the same irrespective during which calendar month the claim spell has started. This assumption is likely violated in our application because winter and summer unemployment in the German labour market have different patterns. In order to relax this restriction, model (3) is modified by allowing the density to vary with calender month of unemployment benefit entry. This is achieved by adopting another idea from actuarial sciences (compare Spierdijk and Koning, 2014). In particular, we use claim duration density estimates that are obtained from a duration model. In order to allow for seasonality in claim durations the Kaplan-Meier estimator (Kaplan and Meier, 1958) is applied to samples that are stratified by calendar month of the claim's start date. Thus, this estimator requires individual level benefit claim duration data. For more details on this approach compare Appendix A.I. The resulting density estimate is $f^{K M}(l, j)$, where $l$ is again the claim duration and $j=1, \ldots, 12$ is the calendar month of the start date. The third forecast for $\mathrm{A}$ is then obtained by

$$
O_{t}^{K M(A)}=\sum_{i=1}^{N_{t 0}} c f^{K M}\left(l_{i}(t), j_{i}\right),
$$

where $c f^{K M}$ is again the conditional density and analogously defined as in model (3). Going from $O_{t}^{C L(A)}$ to $O_{t}^{K M(A)}$ therefore corresponds to changing the estimator for the conditional densities. While allowing for seasonality patterns in the distribution of the duration of claim spells is a useful extension (this is also considered by Lee et al., 2015, in the context of structured density forecasting), there is another restriction that is likely not to hold. So far the density estimates do not vary in calendar time. This implies that beside seasonal effects, the densities are not allowed to vary with business cycle or may not be driven by a trend. The fourth 
forecast relaxes this restriction. This is done by partly parameterizing the duration model. In particular, the semiparametric Cox proportional hazard duration model (Cox, 1972) is used to model the conditional densities. For more details on the Cox model compare Appendix A.I. A linear trend component $t$ is the parametric part. Adding a trend makes is possible for predicted densities to be different from past densities, thus allowing for possible general behavioural or macroeconomic changes over time. Estimation is also stratified by calendar month of the claim start date as in the case of the Kaplan-Meier estimator. The resulting density is then $f^{C o x}(l, j, s)$, where $l$ is the claim duration, $j$ is the calendar month of the start date and $s$ is the year of the start date (with $s=1, \ldots, 4$ correspond to $2002, \ldots, 2005$, respectively). Thus, the fourth forecast is obtained by

$$
O_{t}^{C o x(A)}=\sum_{i=1}^{N_{t 0}} c f^{C o x}\left(l_{i}(t), j_{i}, s_{i}\right)
$$

where $c f^{C o x}$ is analogously defined as in (3). The difference between $O_{t}^{K M(A)}$ and $O_{t}^{C o x(A)}$ is therefore that the latter allows the underlying densities to have a trend in calendar time due to for example the business cycle, while the former restricts them to be calendar time invariant other than the seasonality effects.

Forecast B The next step is to create forecasts for the number of benefit claimants leaving benefits in 2006 who have started their claim periods after Dec 2005. For this purpose $I_{t}^{T S}$ is used as a prediction for the number of benefit inflows in period $t$, where $t=49, \ldots, 60$ (calendar year 2006). The forecasted number of exits in period $t+l$ of those entering benefits in period $t$ is then $O_{t, t+l}^{M(B)}=I_{t}^{T S} * f^{M}(l, j, s)$, where $M=\{C L, K M, C O X\}, j$ is the calendar month of entry, and $s$ is the start year of entry ( $=5$ for 2006$)$. The density estimates $f^{M}$ are the same as in forecasts A. Since all these claimants enter benefits in 2006, the conditional density 
corresponds to the marginal density. $f^{C o x}(l, j, 5)$ is an extrapolation using the estimated trend pattern of the pervious years. Based on these forecasts, $O_{t}^{M(B)}$ with $M=\{C L, K M, C O X\}$ is obtained by

$$
O_{t}^{M(B)}=\sum_{r \leq t} O_{r, t}^{M(B)}
$$

for $t=49, \ldots, 60$. We therefore obtain three forecasts for each month in 2006 which are due to different models for the estimated density of claim duration.

The total number of forecasted outflows out of unemployment insurance is $O_{t}^{M}=O_{t}^{M(A)}+O_{t}^{M(B)}$ with $M=\{C L, K M, C O X\}$. The quality of the forecasts is assessed by considering absolute prediction errors for each calendar month of 2006 and for the entire year 2006. Moreover, bootstrap confidence intervals, mean squared error (MSE) and mean squared forecast error (MSFE) for selected forecasts are reported. However, the MSE for forecast B is computed for given $I_{t}^{T S}$, thus ignoring the uncertainty induced by the time series forecast for the inflows.

Stata code for the various analysis models, except for the Chain Ladder Model, is provided as supplementary material. The results for the Chain Ladder model have been obtained with $\mathrm{R}$ using sample code that will be released by L.Martínez as a package "Double Chain Ladder". Extracts of aggregated data such as the time series, the data triangle and resulting densities are also made available in the supplementary material. They can be used to construct the various forecasts without having access to the SIAB.

\section{Empirical Results}

This section presents estimation results when the methods of the previous section are applied to German administrative labour market data as described in Section 3. Figure 1 presents 
Figure 1: Time-series of unemployment insurance out- and inflows with time series forecasts and their $95 \%$ confidence intervals for 2006 .
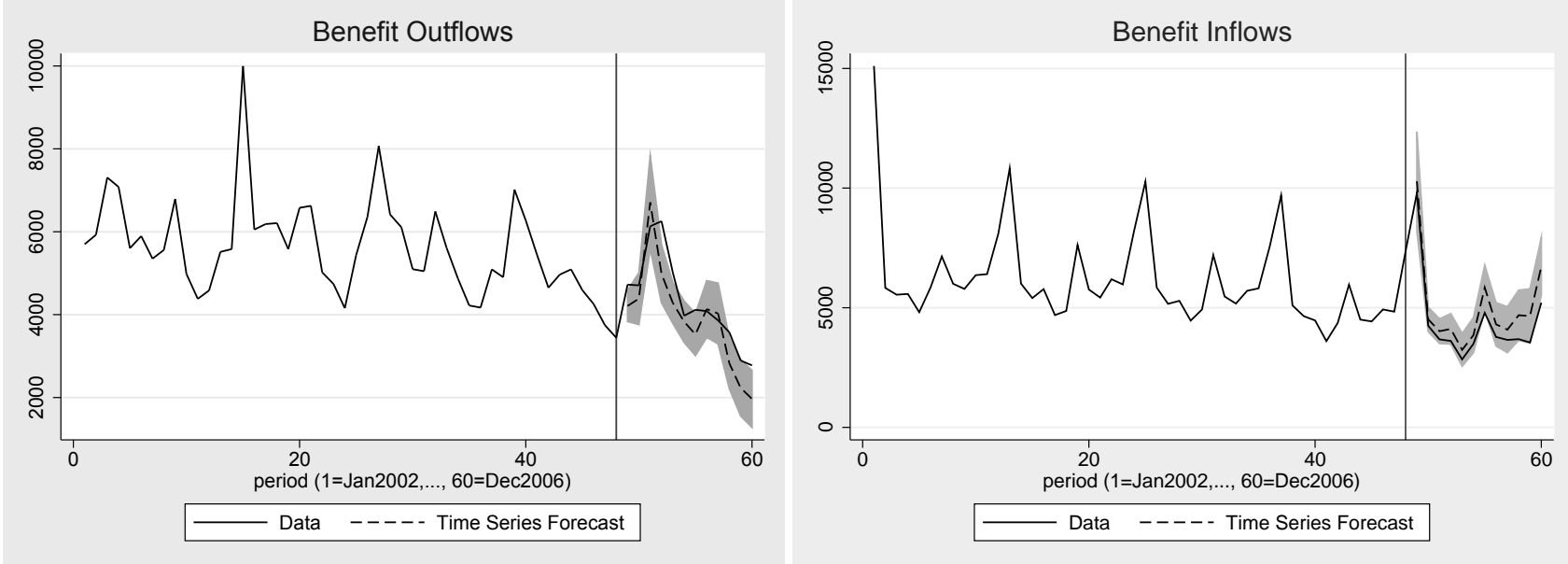

the time series of unemployment insurance claim outflows and inflows for the period Jan2002-

Dec2006. It also shows the time series forecasts for the year 2006 obtained from models (1) and

(2) with their asymptotic $95 \%$ confidence intervals. It is apparent that these extrapolations fit the observed data pretty well. For completeness full estimation results for the time series forecasts with confidence intervals, MSE and MSFE are reported in Table 3 in the Appendix. The remainder of this section compares the various micro analysis based forecasts discussed in the previous section with the time series forecast of model (1). We begin with forecasts A, followed by $\mathrm{B}$ and $\mathrm{A}+\mathrm{B}$. The first step is to apply the CLM. Resulting estimated densities for the distribution of benefit inflows and benefit claim duration (compare Equation (6) in the Appendix) are shown in Figure 2. The estimated density of inflows captures well the observed inflow pattern (as presented in Figure 1) except for the last couple of months in 2005, where the CLM density estimate continues to decline but the actual number of outflows increases (compare Figures 1). This discrepancy could be due to that the data triangle for the CLM (as shown in Figure 4) does not include information on seasonally unemployed during the winter 2005/06. These individuals start their winter unemployment at year end of 2005 and do not return to employment before spring 2006. The estimated density for the distribution of the 
length of claim periods is generally declining with duration but has several spikes. These peaks are located at 12, 22, 26 and 32 months and are also visible in the observed data triangle in Figure 4. They correspond to the maximum benefit entitlement length for some groups of unemployed that fully exploit their entitlements. Unemployed younger than age 45 had a maximum entitlement length of 12 months while older unemployed were entitled to up to 32 months depending on their employment history. For comparison a pooled Kaplan-Meier estimate for the same density is obtained. This estimate uses all claim durations starting between 2002 and 2005 and censors them at the end of 2005. This estimate was found to be very similar to the CLM estimate and is therefore not presented. Instead, Figure 3 shows Kaplan-Meier estimates for the same period but stratified by calendar month. These are the $f^{K M}$ that are used to construct $O_{t}^{K M}$. It is apparent that the density of claim duration varies with season. For example the densities for April $(=4)$ and December $(=12)$ look rather different. We shall therefore expect that this additional flexibility in the model that is not captured by CLM will contribute to an improvement of the forecast. In contrast, plots for the estimated densities on the grounds of the Cox proportional hazard model are not presented because they turned out to be of rather similar shape as the KM estimates in Figure 3.

We have now all ingredients together to construct the various forecasts A which are reported in Table 1. For validation reasons the table also contains the actual data for 2006. It is apparent from this table that the CLM forecast is systematically too low. The other three models (CL, KM and Cox) produce forecasts which are much closer to the observed numbers. The poor performance of CLM in this application is likely due to the inaccuracy of the estimated inflows during the final months of 2005. The downward bias in the estimated density of entries causes the downward bias in the forecasted outflows, in particular during spring 2016. Thus going 
Figure 2: Estimated densities: Chain Ladder Method.
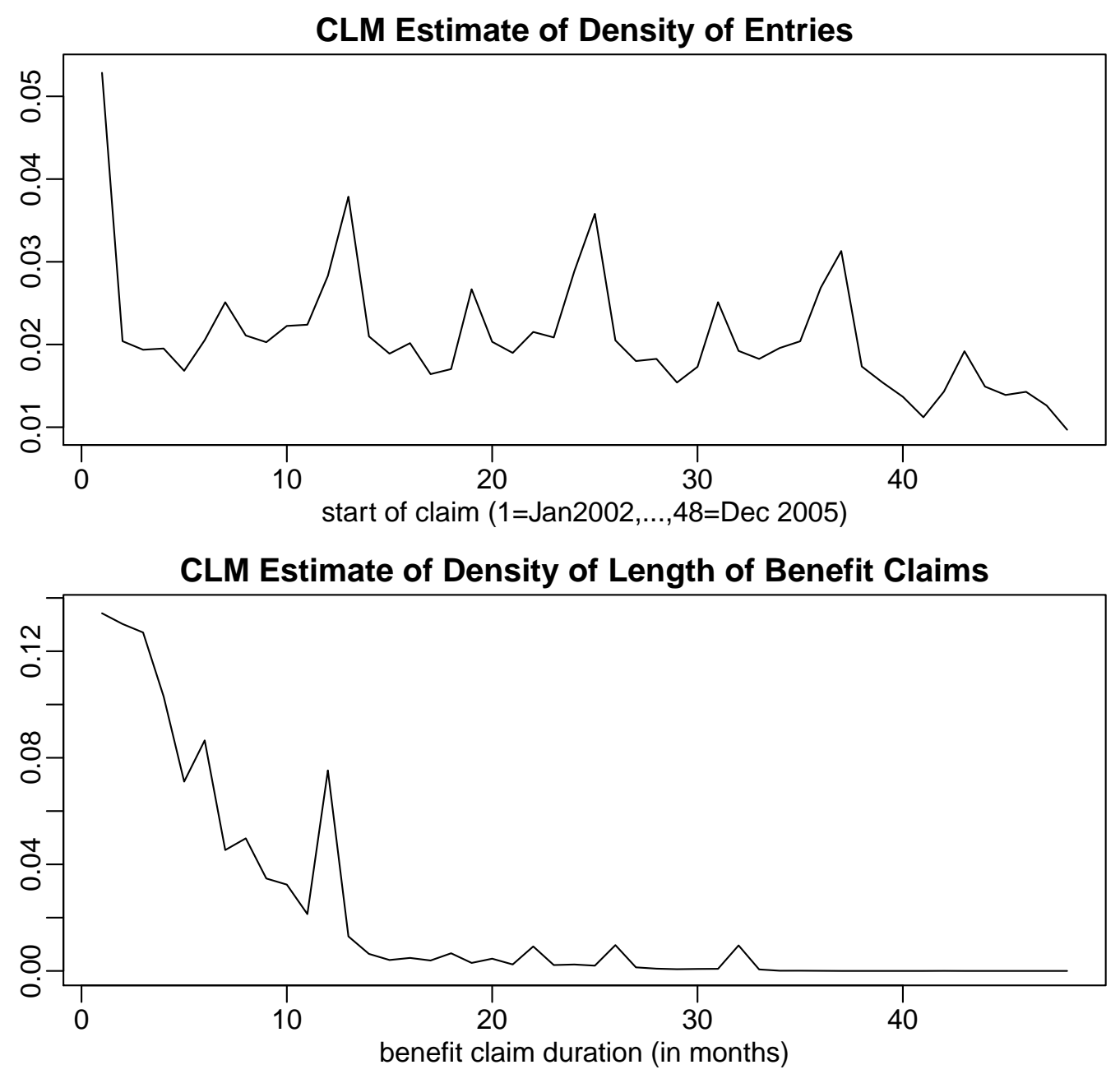
Figure 3: Densities of length of benefit claim duration stratified by month: Kaplan-Meier estimator. Start of claiming benefits: 2002-2005.

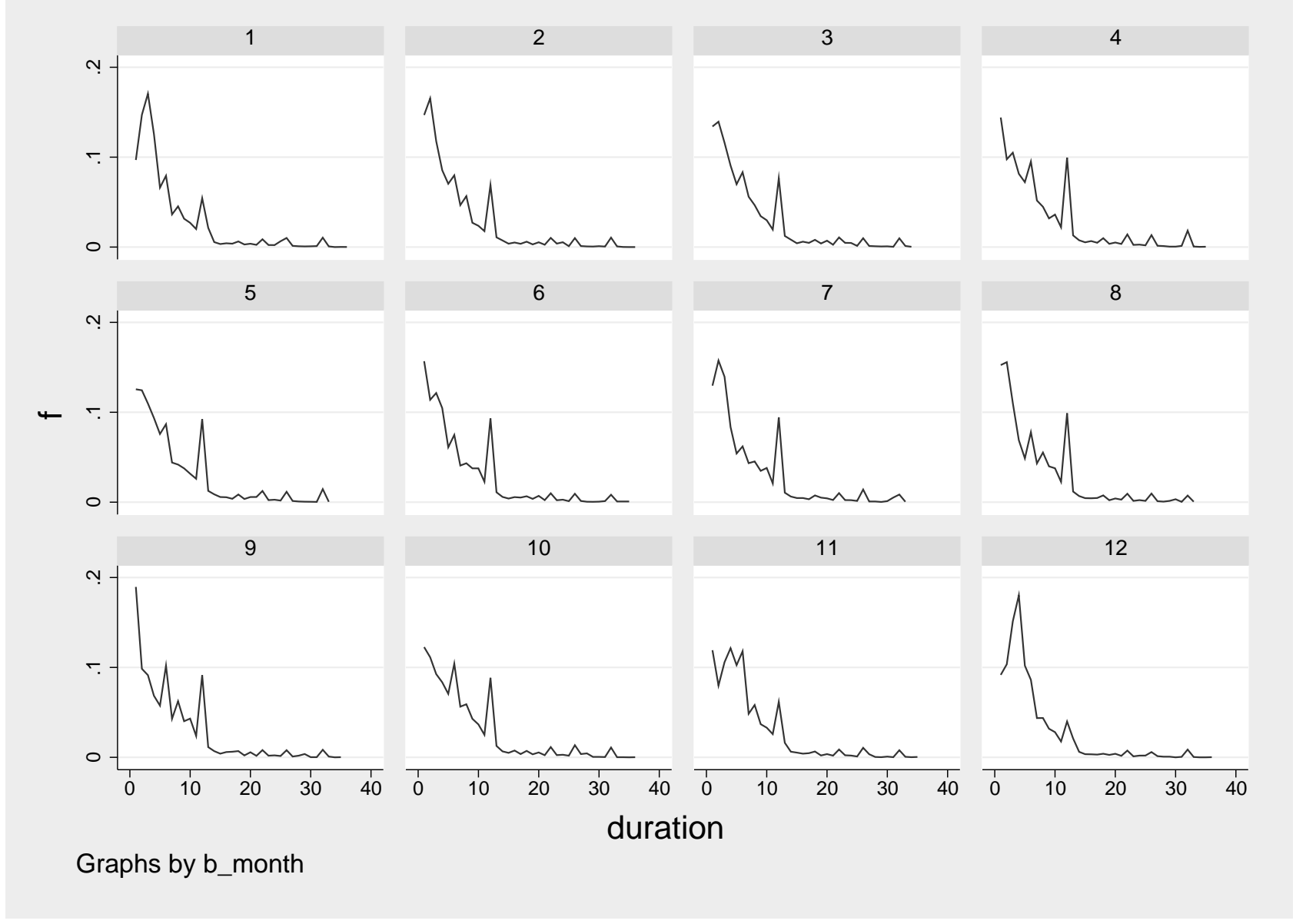


from the simple triangle data on outflows in past periods (to construct $O_{t}^{C L M(A)}$ ) to using data on the stock of benefit claimants at the end of 2005 (to construct $O_{t}^{C L(A)}$ ) results in an improved estimate (smaller forecast errors). It is also apparent that the forecast improves further when using stratified nonparametric KM estimates (to obtain $O_{t}^{K M(A)}$ ) instead of the pooled CL density. But this improvement is only slight. Using the Cox model (to obtain $O_{t}^{\operatorname{Cox}(A)}$ ) strongly improves the total forecast for the year 2006 but does not improve monthly forecasts on average. The CM, KM and Cox forecasts have a rather small prediction error. For example the Cox model based forecast is too low by only 270 units. This is a small number for around $27 \mathrm{~K}$ outflows to be predicted. Table 4 in the Appendix reports $95 \%$ bootstrap confidence intervals for $O_{t}^{C o x}(A)$. These intervals are narrow because the sample of durations consists of more than $350 \mathrm{~K}$ observations. Thus the large number of observation used for the estimation of the individual level estimates leads to a high precision of the latter.

Table 1: Forecasts A: Outflows of those claiming unemployment insurance at the end of 2005.

\begin{tabular}{rrrrrrrrrr}
\hline period & data & $O_{t}^{C L M(A)}$ & error $^{\natural}$ & $O_{t}^{C L(A)}$ & error $^{\natural}$ & $O_{t}^{K M(A)}$ & error $^{\natural}$ & $O_{t}^{\operatorname{Cox}(A)}$ & error $^{\natural}$ \\
\hline jan-06 & 3546 & 3637 & 91 & 5070 & 1524 & 4253 & 707 & 3841 & 295 \\
feb-06 & 4645 & 3073 & 1572 & 4320 & 325 & 4097 & 548 & 3713 & 932 \\
mar-06 & 3846 & 2490 & 1356 & 3612 & 234 & 4082 & 236 & 3745 & 101 \\
apr-06 & 3990 & 2052 & 1938 & 2930 & 1060 & 3447 & 543 & 3252 & 738 \\
maj-06 & 2462 & 1793 & 669 & 2506 & 44 & 2642 & 180 & 2563 & 101 \\
jun-06 & 2161 & 1557 & 604 & 2147 & 14 & 2238 & 77 & 2219 & 58 \\
jul-06 & 1519 & 1308 & 211 & 1742 & 223 & 1781 & 262 & 1805 & 286 \\
aug-06 & 1472 & 1129 & 343 & 1509 & 37 & 1528 & 56 & 1581 & 109 \\
sep-06 & 1039 & 942 & 97 & 1271 & 232 & 1259 & 220 & 1323 & 284 \\
okt-06 & 955 & 790 & 165 & 1081 & 126 & 991 & 36 & 1057 & 102 \\
nov-06 & 810 & 635 & 175 & 1014 & 204 & 843 & 33 & 946 & 136 \\
dec-06 & 687 & 402 & 285 & 742 & 55 & 712 & 25 & 817 & 130 \\
average & & & 626 & & 340 & & 244 & & 273 \\
total 2006 & 27132 & 19808 & 7324 & 27944 & 812 & 27873 & 741 & 26862 & 270 \\
\hline
\end{tabular}

घ: Absolute value

$\ddagger$ : Average of absolute monthly errors 
Table 2 presents the forecasts $\mathrm{B}$ and $\mathrm{A}+\mathrm{B}$ for the different models and the observed outflows in 2006. Two time series forecasts are presented. One resulting from Model (1), and an intercept corrected forecast. The time series based forecasts for $\mathrm{A}+\mathrm{B}$ have the smallest prediction error among all contenders (if we exclude the last two columns which will be discussed below). This is because they have both the smallest average monthly absolute error and the smallest absolute yearly error. Among the micro data based models, the forecast based on the Cox proportional hazard model performs best, followed by the stratified KM estimator based model and the CL density based model. Thus, the increased flexibility in the micro based model by allowing for seasonality in densities and trend extrapolation of the densities leads to a reduction in the forecast error. Although, the time series forecasts have a slightly smaller errors than $O_{t}^{C o x}$, the difference is small relative to the size of the total numbers to be forecasted. When looking in more detail at the errors of the KM and the Cox based forecasts it becomes apparent that the quality of these forecasts deteriorates the more distant the prediction is. In contrast, there is no evidence for such a pattern in the time series forecast. For instance the average absolute monthly error for the first six months of 2006 is 594 for the time series model (1) whereas it is only 493 for the KM density based micro model and 317 for the Cox based density model. Indeed, when we compare the forecasts in Table 1 with the forecasts of the total outflows in Table 2, it also becomes evident that the latter have a considerably larger prediction error. Although, the error of forecast B is not reported it is apparent that the errors of the total $(\mathrm{A}+\mathrm{B})$ forecasts are at least twice as large as the errors of forecasts $\mathrm{A}$ (and sometimes much larger than that). Thus, the micro based models perform rather poorly when they predict future outflows of those who are yet to start a claim. In order to investigate this further, the forecast for the Cox density based model is also constructed using the actual future inflows $\left(I_{t}\right)$ rather than the time series estimates $\left(I_{t}^{T S}\right)$. This forecast and the resulting error are reported 
in the last two columns of Table 2. A direct comparison of the resulting forecasts allows an assessment how the error in $I_{t}^{T S}$ affects $O_{t}^{C o x}$. The average monthly absolute error for the model with $I_{t}$ is 465 , which is around $30 \%$ lower than the equivalent error for $O_{t}^{\text {Cox }}$ (which is 656). A similar pattern is observed for the error of the total number of outflows in 2006 which reduces from 6596 to 3337 . A decline of $50 \%$. Thus, the increase in the size of the error in $O_{t}^{C o x}$ in the distance of the forecast period is to a larger extent due to the error in $I_{t}^{T S}$. This is despite that the latter does not even seem to be too bad (compare Figure 1). Finally, we look at some alternative measures for the quality of the forecasts $O_{t}^{T S}$ and $O_{t}^{C o x}$ which also take higher moments into account. In particular, Tables 3 and 4 in the Appendix report the MSE and MSFE for $O_{t}^{T S}$ and $O_{t}^{C o x}$ respectively. The MSE and MSFE for the Cox model based forecast are considerably smaller for the first half of the year. For instance for February 2006 the time series forecast error is 100 lower but the associated MSE is $11 \%$ greater. This reflects the observation that the confidence intervals for the micro data based forecast have only a width of around 100 units, while the time series forecast's confidence intervals are much wider (more than 1000 units). This is due the individual level data model relies on more than 350K observations while the time series model only uses 48 observations. The MSFE for the Cox model based forecast for the first 6 months is smaller than the MSFE for the two time series forecasts. However, for the second half of the year, the forecast errors for $O_{t}^{C o x}$ becomes so much larger that the time series forecasts are characterised by a smaller MSE and MSFE. In addition to comparing the MSFE, an associated statistical test, the Diebold-Mariano test, has been conducted to asses whether forecast quality differs statistically. For the entire year 2006 it is found that the intercept corrected time series forecast performs statistically better than the non corrected time series forecast, while there is no statistical difference between these two forecasts and the Cox model based forecast. When only considering the first six months of 
2006 it is found that the statistical difference between the two time series forecasts persists but the Cox model based forecast is significantly better than the intercept corrected time series forecast. Whether the Diebold-Mariano or related tests are indeed applicable to evaluate the performance of the different forecasts is unclear. This is because the asymptotic distribution of the test statistics is obtained by comparing different time series estimates and by letting the number of periods going to infinity.

Robustness checks and policy changes As mentioned at the start of this section, several robustness checks with regard to the specification of the time series model have been conducted. Overall, the reported results were found to be rather representative and for this reason they have been chosen. The analysis has been also repeated to construct forecasts for several other calendar years $(2002,2003,2005,2009)$. Similar result patterns have been obtained for the years 2003-2006 (compare supplementary material Tables S1.-S3). This is a period with stable entitlement rules for unemployment benefits. As indicated in Section 3, several significant policy changes have become effective in the late 1990s, 2006 and 2008 which altered entitlement lengths for unemployment benefits. As a consequence, there were considerable changes in benefit and unemployment duration (compare Arntz et al., 2014 and Lo et al., 2017). This can be also seen in Figure S.1 (supplementary material). For those entering benefits in January 2016, the maximum entitlement length is 32 months. For those entering between February and July, there are hardly any benefit durations longer than 18 months. And from July the maximum increases again to 24 months (as the maximum entitlements were subsequently increased from 18 to 24 months when the benefit reform in 2008 became effective). As a result, the estimation of the claim durations would need to explicitly model these changes, which has not been done. This is also of relevance for the analysis of the year 2006. In particular, forecast B utilises 
estimated claim durations of the pre 2006 system (based on data from the period 2002-2005) but the new entrants from February 2006 possess entitlements according to the new system. Given that the changes in entitlements mainly affected extended benefit durations $>12$ months, the implied misspecification should not have a large effect on the forecasts because those who enter benefits from February 2006 have a benefit duration of less than 12 months at the end of the forecast period (December 2006). However, a bias in estimated claim durations is likely the source of rather different results patterns that have been obtained for the years 2002 and 2009 (compare supplementary material Tables S.4 and S.5). In particular, the time series forecasts are characterised by a lower forecast error and even the use of $I_{t}$ instead of $I_{t}^{T S}$ to construct $O_{t}^{C o x}$ does not lead to a systematic reduction in forecast errors. This highlights the importance of correctly specifying the distribution of claim duration and that any not captured structural changes in these distribution may severely affect the micro data based forecasts.

\section{Summary and recommendations}

This study presents several approaches to modelling macroeconomic forecasts on the basis of individual level statistical estimates. By adopting ideas from actuarial sciences and new developments in statistical theory for in-sample forecasting, it is shown that micro analysis based macro forecasts constitute an interesting alternative to econometric forecasts based on aggregate or time series data. The results for forecasting outflows from unemployment insurance claims in Germany are encouraging. In particular, the forecast quality of the suggested models is found to be better for forecasts of up to around 6 months. It is also found that the size of the forecast error of the micro estimates based forecasts increases quickly for forecasts more than 6 months ahead. This is explained by the underlying estimates on micro level not being able 
to appropriately accommodate future labour market dynamics. It would be a useful extension to incorporate additional micro based forecasts for other labour market transitions (such as benefit inflows). Structured density forecasts for benefit inflows would then replace the time series based forecast to obtain a more precise forecast for the number of future benefit claimants. Despite that forecasts have only been presented for one year, the main result patterns have also been found for other periods with stable unemployment benefit entitlement rules during the 2000s. It is, however, also found that the precision of the suggested forecasts tend to be worse when important policy changes have been implemented which lead to significant changes in entitlement lengths for benefits and therefore in benefit durations. In order to address this, one would need to incorporate additional individual level covariates in the models for the structured density forecasts. These additional factors such as individual benefit entitlement lengths would contribute to a better fit and a bias reduction in estimated densities. This would, however, not come without costs. Enhanced data requirements make it more complicated to implement and run these models in real business operations as data on all variables needs to be available promptly. Similar to Spierdijk and Koning (2014) another extension could be made by adopting a duration model with frailty to allow for unobserved factors in the analysis.

\section{References}

[1] Antonio, K. and Plat, H. (2014). Micro-level stochastic loss reserving in general insurance, Scandinavian Actuarial Journal, 7, 649-669.

[2] Arntz, M., Lo, S.M.S. and Wilke, R.A. (2014). Bounds Analysis of Competing Risks : A Non-parametric Evaluation of the Effect of Unemployment Benefits on Migration, Empirical Economics, 146, 199-228. 
[3] Barnichon, R. and Nekarda, C.J. (2012). The Ins and Outs of Forecasting Unemployment: Using Labor Force Flows to Forecast the Labor Market, Brookings Papers on Economic Activity, Economic Studies Program, The Brookings Institution, 45(2 (Fall)), 83-131.

[4] Brown, L. and Moshiri, S. (2004). Unemployment variation over the business cycles: a comparison of forecasting models, Journal of Forecasting, 23(7), 497-511.

[5] Cox, D.R. (1972). Regression models and life-tables (with discussion), Journal of the Royal Statistical Society, Series B, 34, 187-220.

[6] Elsby, M., Hobijn, B. and Sahin, A. (2015). On the importance of the participation margin for labor market fluctuations. Journal of Monetary Economics, 72, 64-82.

[7] Hiabu, M., Martínez-Miranda, M.D., and Nielsen, J.P. (2014). In-sample forecasting with local linear survival densities, Biometrika, under revision.

[8] Hutter, C. and Weber, E. (2015). Constructing a new leading indicator for unemployment from a survey among German employment agencies, Applied Economics, 47, 3540-3558.

[9] Hutter, C. and Weber, E. (2017). Mismatch and the Forecasting Performance of Matching Functions, Oxford Bulletin of Economics and Statistics, 79, 101-123.

[10] Kalbfleisch, J.D. and Prentice, R.L. (2002). The Statistical Analysis of Failure Time Data, 2nd ed., Wiley Series in Probability and Statistics, Wiley.

[11] Kaplan, E. L., and Meier, P. (1958). Nonparametric estimation from incomplete observations, Journal of the American Statistical Association, 53, 457-481.

[12] Kuang, D., Nielsen, B., and Nielsen J.P. (2009). Chain-Ladder as Maximum Likelihood Revisited, Annals of Actuarial Science, 4, 105-121. 
[13] Kuang, D., Nielsen, B., and Nielsen, J.P. (2011). Forecasting in an Extended Chain-LadderType Model, Journal of Risk and Insurance, 78(2), 345-359.

[14] Lee, Y.K., Mammen, E., Nielsen, J.P. and Park, B.U. (2015). Asymptotics for In-Sample Density Forecasting, Annals of Statistics, 43(2), 620-651.

[15] Lo, S.M.S., Stephan, G. and Wilke, R.A. (2017). Competing Risks Copula Models for Unemployment Duration: An Application to a German Hartz-Reform. Journal of Econometric Methods, 6 .

[16] Mammen, E., Martínez-Miranda, M.D., and Nielsen, J.P. (2015). Structured Density Forecasting with Applications to Non-life Insurance and Mesothelioma Mortality, Insurance: Mathematics and Economics, 61, 76-86.

[17] Martínez-Miranda, M.D., Nielsen, B., and Nielsen, J.P., (2015) Inference and forecasting in the ageperiodcohort model with unknown exposure with an application to mesothelioma mortality, Journal of the Royal Statistical Society, Series A, 178(1), 29-55.

[18] Martínez-Miranda, M.D., Nielsen, J.P., Sperlich, S., and Verrall, R. (2013). Continuous Chain Ladder: Reformulating and generalizing a classical insurance problem, Expert Systems with Applications, 40(14), 5588-5603.

[19] Newey, W.K. and West, K.D. (1987). A Simple, Positive Semi-definite, Heteroskedasticity and Autocorrelation Consistent Covariance Matrix, Econometrica, 55(3), 703-708.

[20] Pigeon, M., Antonio, K., and Denuit, M. (2013). Individual loss reserving with the multivariate skew normal framework, Astin Bulletin, 43(3), 399 - 428. 
[21] Pigeon, M., Antonio, K., and Denuit, M. (2014). Individual loss reserving using paidincurred data, Insurance: Mathematics and Economics, 58(C), 121-131.

[22] Plaßmann, G. (2002) Der Einfluss der Arbeitslosenversicherung auf die Arbeitslosigkeit in Deutschland. Beiträge zur Arbeitsmarkt- und Berufsforschung, 255, Institut für Arbeitsmarkt- und Berufsforschung der Bundesanstalt für Arbeit (IAB), Nuremberg.

[23] Sermpinis, G., Stasinakis C., Theofilatos K. and Karathanasopoulos A. (2014). Inflation and Unemployment Forecasting with Genetic Support Vector Regression, Journal of Forecasting, 33, 455-470.

[24] Spierdijk, L. and Koning, R.H. (2014). Estimating Outstanding Claim Liabilities: The Role of Unobserved Risk Factors, Journal of Risk and Insurance, 81(4), 803-830.

[25] vom Berge, P., Burghardt, A. and Trenkle, S. (2013). Sample of integrated labour market biographies * regional file 1975-2010 (SIAB-R 7510), FDZ-Datenreport, 09/2013 (en), Nuremberg.

[26] Weindorfer, B. (2012). A practical guide to the use of the chain-ladder method for determining technical provisions for outstanding reported claims in non-life insurance. Working Paper, 77/2012, University of Applied Sciences Vienna. 


\section{Appendix}

\section{AI: Statistical Models}

This appendix outlines the various analysis models: Chain Ladder Method and the duration models (Kaplan-Meier, Cox proportional hazard). The data are unemployment benefit spell records for a random sample of individuals and is available for periods $t=1, \ldots, T$, which are 48 months in the application. From these data we can easily compute aggregated information such as the number of individuals starting a benefit claim spell in $t\left(I_{t}\right)$, and the number of benefit leavers in period $t\left(O_{t}\right)$. It is also straightforward to extract semi-aggregated information such as the number of benefit leavers in $t$ with claim duration $l=1, \ldots, T$ and $t+l-1 \in\{1, \ldots, T\}$, denoted by $O_{t l}$. The latter are required for the chain ladder method.

Chain Ladder Method (CLM) A formal overview and presentation of the statistical model can be for example found in Kuang et al. (2009). The CLM bases on semi-aggregated data $O_{t l}$ that can be organised in a matrix $O=\left\{O_{t l}, \forall(t, l) \in \mathcal{I}\right\}$ where $\mathcal{I}=\{(t, l): t$ and $l$ belong to $(1, \ldots, T)\}$ with $t+l-1 \in\{1, \ldots, T\}$. Period $T$ is the edge of the observation period which corresponds to $t=48$ in the application. Thus, only a triangle of this matrix is observable, which is illustrated in Figure 4 for the data that is used in the application in Section 5.

The number of benefit leavers in $t, O_{t}$, can be computed from this matrix by summing up the $O_{t l}$ over the available $l$. It is worth mentioning that $O$ does not include any information on benefit recipients which have not ended their benefit claim spell by period $T$. The CLM is a tool to obtain a forecast for the number of future exits out of benefits for those who are 
Figure 4: Observed data triangle: $O_{t l}$
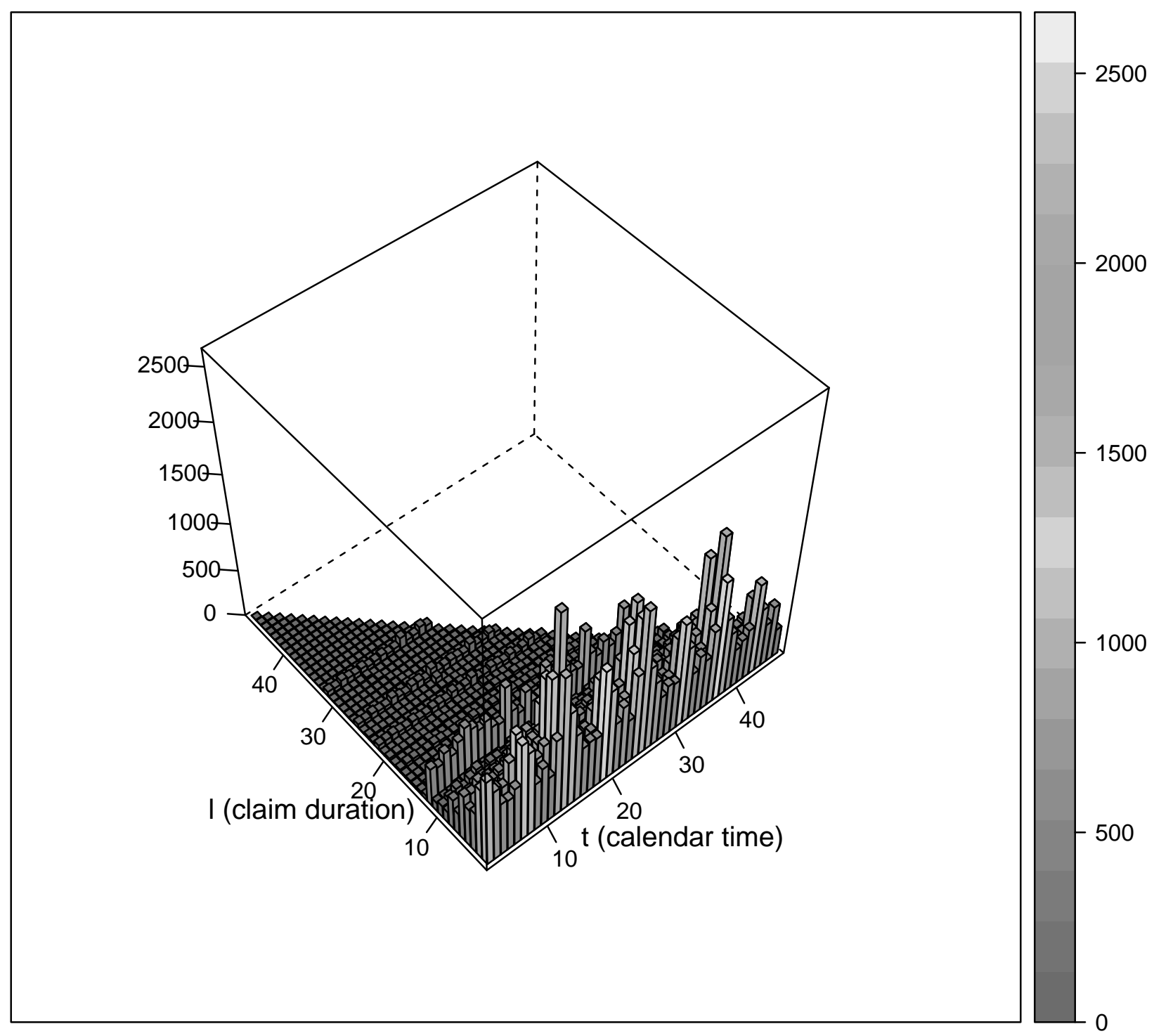
claiming benefits in $T$. This is forecasting $O_{t l}^{(A)}$ for all $t$ and $l$ such that $t+l-1>T$. Evidently, $O_{t l}^{(A)}=O_{t l}$ for $t, l \in \mathcal{I}$. In actuarial sciences this method is used to obtain a forecast of the outstanding liabilities for those contracts which have been already signed. In what follows a short presentation of the underlying stochastic model is provided. $O_{t l}^{(A)}$ can be thought of as a random variable with $E\left(O_{l t}^{(A)}\right)=\kappa_{t} \tau_{l}$ for $t, l=1, \ldots, T . \kappa_{t}$ and $\tau_{l}$ are the unknown parameters of this regression model. This is a simplified model of a more general overparametrised regression problem which is only identified under restrictions on the parameters (compare Kuang et al., 2009). For an intuitive discussion of the underlying regression problem see Martinez et al. (2013). The goal is to estimate $\kappa_{t}$ and $\tau_{l}$. Estimation is by maximum likelihood using the following model:

$$
\log \left\{E\left(O_{t l}^{(A)}\right)\right\}=\mu_{t l}=\gamma_{t}+\delta_{l}
$$

with $\gamma_{t}=\log \kappa_{t}, \delta_{l}=\log \tau_{l}$ and let $\eta=\left(\gamma_{1}, \ldots, \gamma_{T}, \delta_{1}, \ldots, \delta_{T}\right) \in \mathbb{R}^{2 \mathrm{~T}}$. By assuming that $O_{t l}^{(A)}$ are independent for $t, l \in \mathcal{I}$ and that $O_{l t}^{(A)}$ is a Poisson distributed count variable with

$$
P\left(O_{t l}^{(A)}=y\right)=\frac{\exp \left(\mu_{t l} y\right)}{y !} \exp \left\{-\exp \left(\mu_{t l}\right),\right\}
$$

the log-likelihood function of the model is

$$
L(\eta)=\sum_{t, l \in \mathcal{I}}\left\{\mu_{t l}(\eta) O_{t l}-\exp \left(\mu_{t l}(\eta)\right)-\log \left(O_{t l} !\right)\right\}
$$

The maximum likelihood estimator for $\eta$ is obtained by maximising $L$. The CLM can be therefore seen as a maximum likelihood estimator that is consistent under the usual restrictions. From the estimated coefficients it is straightforward to compute estimated $O_{t l}^{(A)}$, denoted as $O_{t l}^{C L M(A)}$, and $O_{t}^{C L M(A)}$. The resulting $O_{t l}^{C L M(A)}$ for the data from Section 5 are displayed in Figure 5 for illustration.

The densities for the entry into claiming benefits and the density of claim duration can be easily 
Figure 5: CLM forecasts $O_{t l}^{C L M(A)}$

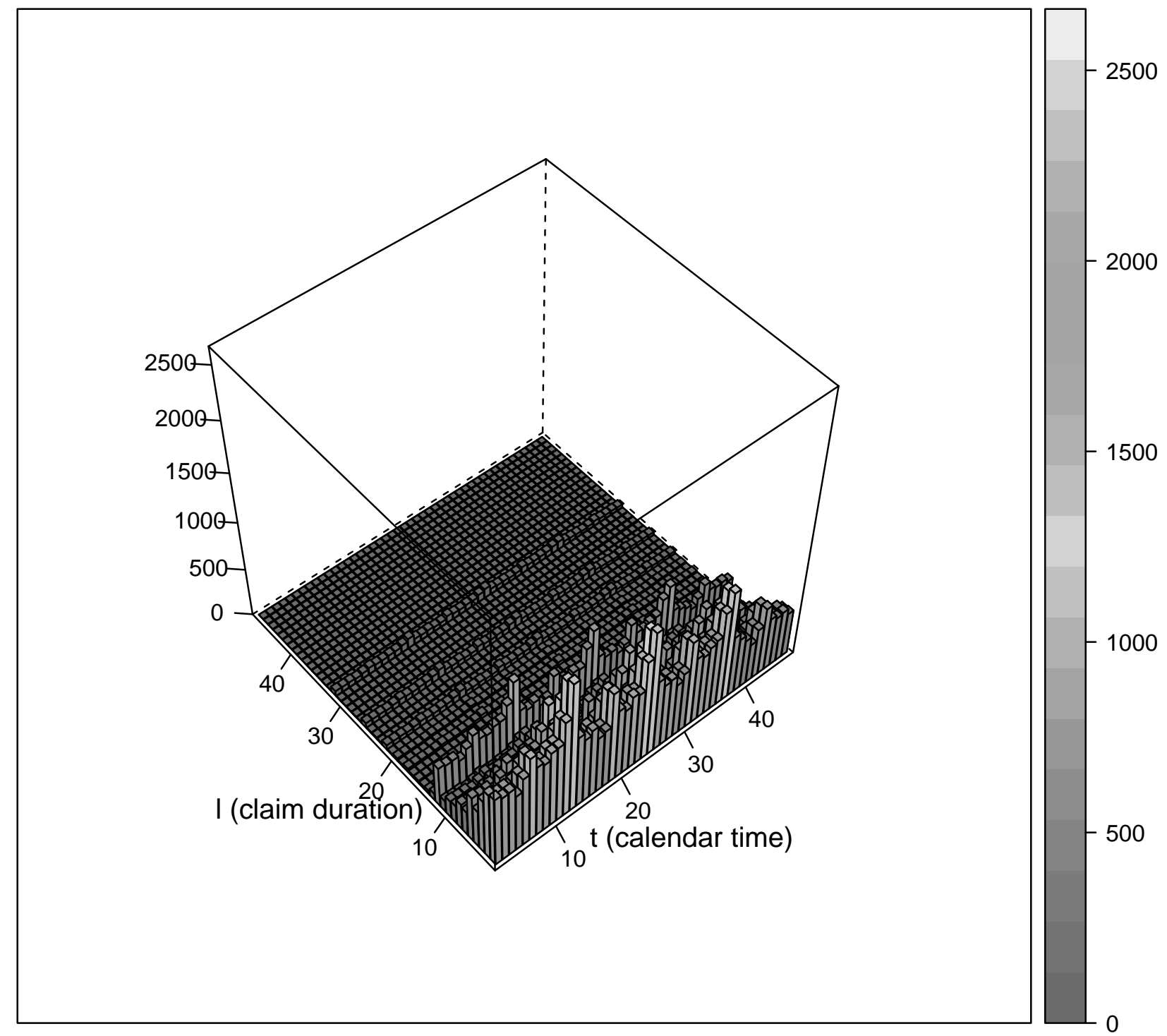


determined from the estimated $\eta$ by normalisation. For example the density of claim duration is obtained by

$$
f^{C L}(l)=\delta_{l} / \sum_{j=1}^{T} \delta_{j}
$$

for $l=1, \ldots, T$. The estimated densities in the application are shown in Figure 2.

Duration Models These models use individual level duration data and not (semi)-aggregated data. Suppose there are $N$ individuals in the "raw" data that generate in total $i=1, \ldots, J$ benefit claim spells. $J$ is smaller than $N$ in the application because most individuals do not have a claim spell, although some individuals will have multiple claim spells due to repeated unemployment. If a spell is not fully observable, i.e. not finished at period $T$ (December 2005 in the application), it is marked as right censored. The only reason for censoring is the end of the observation period. Duration models are commonly used to estimate the survival function $S(l)=P(L \geq l)$, where $l$ is here the benefit claim duration. There exists a wealth of duration models which impose different restrictions on $S(l)$. In the analysis of Section 5 two flexible models are employed: one nonparametric and one semi-parametric model.

The Kaplan-Meier estimator (Kaplan-Meier, 1958) is a widely used nonparametric estimator. Suppose $l_{1}, \ldots, l_{q}$ are discrete failure times with $q<J$. The estimator is

$$
S^{K M}(l)=\Pi_{s \mid l_{s}<l} \frac{r_{l}-d_{l}}{r_{l}}
$$

with $r_{l}$ is the number of spells at risk at duration $l$ and $d_{l}$ is the number of spells that end at duration $l$. This estimator is consistent in presence of independent right censoring as in the application. The density of claim duration is obtained by $f(l)=-[S(l+1)-S(l)]$. The resulting estimated densities in the application are shown in Figure 3. While being very flexible due to its nonparametric nature, a disadvantage is that it cannot control for covariates such as 
calendar time variables to account for business cycle and seasonality effects. In order to add this feature a semiparametric model, the Cox-proportional hazard model (Cox, 1972), is applied. This model assumes an additive structure for the hazard function given some covariates $x$ :

$$
\lambda(l \mid x)=\lambda_{0}(l) \phi(x)
$$

where $\lambda_{0}$ is a nonparametric function and $\phi(x)=\exp (x \nu)$ with $\nu$ are unknown parameters. In this model we have

$$
\begin{aligned}
S(l \mid x) & =\exp \left(-\int_{0}^{l} \phi(x) \lambda_{0}(s) d s\right) \\
& =\exp \left(-\phi(x) \int_{0}^{l} \lambda_{0}(s) d s\right) \\
& =\left[\exp -\int_{0}^{l} \lambda_{0}(s) d s\right]^{\phi(x)} \\
& =\left[S_{0}(l)\right]^{\phi(x)}
\end{aligned}
$$

with $S_{0}(l)$ is the survival function related to $\lambda_{0}(l)$. The density of claim duration is

$$
f(l \mid x)=-\phi(x)\left[S_{0}(l)\right]^{\phi(x)-1} f_{0}(l)
$$

with $f_{0}(l)$ is the density related to $\lambda_{0}(l)$. The estimated $f^{C o x}$ are easily obtained once $\lambda_{0}(l)$ and $\phi(x)$ have been estimated by partial likelihood. For a full presentation of the likelihood function and estimation by partial likelihood compare for example Chapter 4 in Kalbfleisch and Prentice (2002). 


\section{AII: Tables}

Table 3: Summary table of time series forecast for benefit outflows in 2006.

\begin{tabular}{|c|c|c|c|c|c|c|}
\hline & data & forecast & error & lower $95 \% \mathrm{CI}^{\natural}$ & upper $95 \% \mathrm{CI}^{\natural}$ & $\mathrm{MSE}^{\ddagger}$ \\
\hline jan-06 & 4725 & 4210 & -515 & 3833 & 4586 & $302,193.4$ \\
\hline feb-06 & 4706 & 4385 & -321 & 3760 & 5009 & $204,506.7$ \\
\hline mar-06 & 6131 & 6710 & 579 & 5588 & 7831 & $662,613.2$ \\
\hline apr-06 & 6255 & 4986 & -1269 & 4285 & 5686 & $1,738,139.0$ \\
\hline maj-06 & 5015 & 4282 & -733 & 3790 & 4773 & $600,190.5$ \\
\hline jun-06 & 3974 & 3830 & -144 & 3330 & 4329 & $85,694.2$ \\
\hline jul-06 & 4116 & 3522 & -594 & 3014 & 4029 & $419,987.0$ \\
\hline aug-06 & 4086 & 4135 & 49 & 3450 & 4819 & $124,396.9$ \\
\hline sep-06 & 3861 & 4027 & 166 & 3294 & 4759 & $167,056.8$ \\
\hline okt-06 & 3573 & 2824 & -749 & 2211 & 3436 & $658,798.0$ \\
\hline nov-06 & 2892 & 2232 & -660 & 1553 & 2910 & $555,433.8$ \\
\hline dec-06 & 2776 & 1967 & -809 & 1279 & 2654 & $777,501.2$ \\
\hline \multicolumn{6}{|c|}{$\mathrm{MSFE}^{\dagger}$ for year 2016: } & $408,816.0$ \\
\hline \multicolumn{6}{|c|}{ MSFE $^{\dagger}$ for January-June 2016: } & $478,648.8$ \\
\hline \multirow{2}{*}{\multicolumn{6}{|c|}{$\begin{array}{l}\text { MSFE }^{\dagger} \text { (intercept corrected) for year 2016: } \\
\text { MSFE }^{\dagger} \text { (intercept corrected) for January-June 2016: }\end{array}$}} & $267,843.3$ \\
\hline & & & & & & $345,308.8$ \\
\hline
\end{tabular}

घ: Asymptotic confidence interval derived from OLS regression with robust standard errors.

¥: Mean squared error of forecast for month $t$; defined as $\left(O_{t}-O_{t}^{\text {forecast }}\right)^{2}+\operatorname{var}\left(O_{t}^{\text {forecast }}\right)$.

$\dagger^{\dagger}$ : Mean squared forecast error for year 2006; defined as $(1 / 12) \sum_{t=1}^{12}\left(O_{48+t}-O_{48+t}^{\text {forecast }}\right)^{2}$. 
Table 4: Confidence intervals, MSE and MSFE for $O_{t}^{C o x}$ for the year 2006.

\begin{tabular}{|c|c|c|c|c|c|c|c|}
\hline & \multicolumn{2}{|c|}{$O_{t}^{C o x}(A)$} & \multicolumn{2}{|c|}{$\begin{array}{c}95 \% \mathrm{CI}^{\natural} \text { for } \ldots \\
O_{t}^{\operatorname{Cox}(B)}\end{array}$} & \multicolumn{2}{|c|}{$O_{t}^{C o x}$} & \multirow[t]{2}{*}{$\mathrm{MSE}^{\ddagger}$ for $O_{t}^{C o x}$} \\
\hline & lower & upper & lower & upper & lower & upper & \\
\hline jan-06 & 3788 & 3892 & 425 & 454 & 4224 & 4335 & $198,987.5$ \\
\hline feb-06 & 3662 & 3766 & 1389 & 1451 & 5070 & 5195 & $183,310.1$ \\
\hline mar-06 & 3690 & 3801 & 2351 & 2426 & 6070 & 6190 & $1,076.2$ \\
\hline apr-06 & 3208 & 3299 & 2771 & 2843 & 6004 & 6118 & $38,555.8$ \\
\hline maj-06 & 2519 & 2598 & 2484 & 2554 & 5030 & 5132 & $5,136.7$ \\
\hline jun-06 & 2186 & 2254 & 2494 & 2568 & 4695 & 4800 & $593,682.3$ \\
\hline jul-06 & 1773 & 1834 & 2777 & 2849 & 4571 & 4665 & $248,665.1$ \\
\hline aug-06 & 1548 & 1611 & 3138 & 3219 & 4709 & 4809 & $452,323.3$ \\
\hline sep-06 & 1292 & 1352 & 3742 & 3830 & 5060 & 5164 & $1,555,745.0$ \\
\hline okt-06 & 1032 & 1079 & 3569 & 3652 & 4619 & 4715 & $1,197,436.0$ \\
\hline nov-06 & 920 & 972 & 3101 & 3174 & 4042 & 4130 & $1,421,420.0$ \\
\hline \multirow[t]{2}{*}{ dec-06 } & 790 & 845 & 3186 & 3264 & 3999 & 4081 & $1,605,818.0$ \\
\hline & \multicolumn{6}{|c|}{$\mathrm{MSFE}^{\dagger}$ for year 2016: } & $\begin{array}{l}624,403.0 \\
169208.3\end{array}$ \\
\hline
\end{tabular}

घ: Bootstrap confidence interval.

$\ddagger$ : Mean squared error of forecast for month $t$; defined as $\left(O_{t}-O_{t}^{\text {forecast }}\right)^{2}+\operatorname{var}\left(O_{t}^{\text {forecast }}\right)$.

${ }^{\dagger}$ : Mean squared forecast error for year 2006; defined as $(1 / 12) \sum_{t=1}^{12}\left(O_{48+t}-O_{48+t}^{\text {forecast }}\right)^{2}$.

Note: Bootstrap statistics CI and MSE are derived from random variation in $c f^{C o x}$ for given $I_{t}^{T S}$. 